\title{
A New Isoflavan Glucoside from the Roots of Astragalus
}

membranaceus var. mongholicus

\author{
Yadong Wang $\oplus^{1}$, Chao Wang $\oplus^{2}$, Yuanhao Wang $\oplus^{* 2}$, Qing $\mathrm{Hu} \oplus^{* 2}$ \\ and Fenghuan Wang ${ }^{1}$
}

\author{
${ }^{1}$ School of Light Industry, Beijing Technology and Business University, Beijing 100048, China \\ ${ }^{2}$ SUSTech Engineering Innovation Center, School of Environmental Science and \\ Engineering, Southern University of Science and Technology, Shenzhen 518055, China
}

(Received September 09, 2021; Revised October 18, 2021; Accepted October 24, 2021)

\begin{abstract}
A new isoflavan glucoside, namely astramemside A (1), together with six known compounds (2-7) were obtained from the roots of a traditional Chinese medicine, Astragalus membranaceus var. mongholicus. Their structures were elucidated by spectroscopic analyses (HRESIMS, UV, IR, 1D and 2D NMR), and the absolute configuration of $\mathbf{1}$ was determined by combination of chemical transformation and single-crystal X-ray diffraction. Compound $\mathbf{1}$ showed moderate inhibition on nitric oxide (NO) production induced by lipopolysaccharide in RAW264.7 cells with an $\mathrm{IC}_{50}$ value of $38.98 \pm 5.28 \mu \mathrm{M}$.
\end{abstract}

Keywords: Astragalus membranaceus var. mongholicus; Leguminosae; isoflavan glucoside. () 2021 ACG Publications. All rights reserved.

\section{Plant Source}

In this phytochemical study of the roots of Astragalus membranaceus var. mongholicus (Leguminosae) (Plant materials see supporting information), a new isoflavan glucoside, astramemside A (1), together with six known compounds (2-7) were isolated. Herein, we report the isolation and structural elucidation of these compounds.

\section{Previous Studies}

Astragalus membranaceus var. mongholicus, also known as "Huang-Qi" in traditional Chinese medicine (TCM), is a perennial herb widely distributed in North, Northeast, and Northwest China [1]. Its roots have long been used in TCM for various purposes, such as antiperspirant, antimicrobial, antiinflammatory, diuretic and tonic, etc [2]. Particularly, the extract of this plant can enhance immune system of human [3]. Previous studies on chemical composition of this species showed that isoflavonoids and triterpene saponins were major constituents [4-10]. Some of these constituents

\footnotetext{
*Corresponding author: E- Mail: wangyh2020@mail.sustech.edu.cn (Y. Wang); huq@ sustech.edu.cn (Q. Hu)
} 
exhibited diverse pharmacological properties such as triglyceride accumulation inhibitory and antiinflammatory activities $[4,11]$.

\section{Present Study}

The EtOAc fraction of A. membranaceus var. mongholicus was separated by repeated column chromatography over silica gel, reversed phase $\mathrm{C}_{18}\left(\mathrm{RP}-\mathrm{C}_{18}\right), \mathrm{LH}-20$ gel, and finally HPLC to obtain compounds 1-7 (Figure 1) (detailed separation process see supporting information).

Astramemside A (1): white amorphous powder, $[\alpha]^{25}-30.8(c 0.2, \mathrm{MeCN}) ; \mathrm{UV}(\mathrm{MeCN}) \lambda_{\max }(\log \varepsilon)$ 280 (2.87), 206 (4.11) nm; IR (KBr) $v_{\max }$ 3395, 2921, 2850, 1712, 1619, 1505, 1464, 1171, $1095 \mathrm{~cm}^{-1}$; ${ }^{1} \mathrm{H}$ and ${ }^{13} \mathrm{C}$ NMR data, see Table 1; HRESIMS $m / z 555.1838[\mathrm{M}+\mathrm{Na}]^{+}$(calcd for $\mathrm{C}_{27} \mathrm{H}_{32} \mathrm{O}_{11} \mathrm{Na}^{+}$, 555.1837).

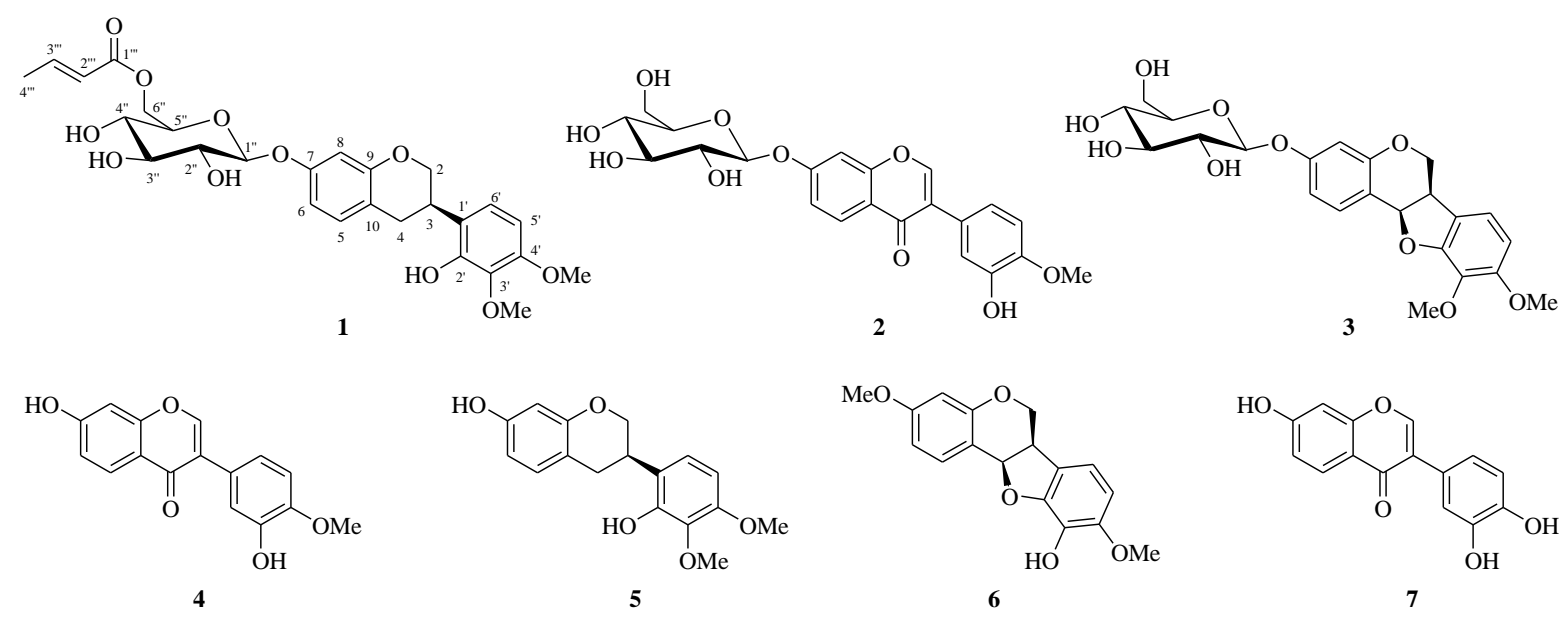

Figure 1. Structures of compounds 1-7

Compound 1, white amorphous powder, displayed a negative optical rotation $\left([\alpha]^{25}-30.8\right.$, in $\mathrm{MeCN}$ ). Its molecular formula, $\mathrm{C}_{27} \mathrm{H}_{32} \mathrm{O}_{11}$, was established by the HRESIMS ion peak at $\mathrm{m} / \mathrm{z} 555.1838$ $[\mathrm{M}+\mathrm{Na}]^{+}$(calcd for $\mathrm{C}_{27} \mathrm{H}_{32} \mathrm{O}_{11} \mathrm{Na}^{+}, 555.1837$ ). In the IR spectrum, the absorption bands of hydroxy $\left(3395 \mathrm{~cm}^{-1}\right)$, carbonyl group $\left(1712 \mathrm{~cm}^{-1}\right)$ and aromatic ring $\left(1619,1505\right.$ and $1464 \mathrm{~cm}^{-1}$ ) were observed. The ${ }^{1} \mathrm{H}$ NMR spectroscopic data (Table 1) displayed signals for a 1,3,4-trisubstituted benzene ring $\left[\delta_{\mathrm{H}} 6.97(1 \mathrm{H}, \mathrm{d}, J=8.3 \mathrm{~Hz}, \mathrm{H}-5), 6.59(1 \mathrm{H}, \mathrm{dd}, J=8.3,2.5 \mathrm{~Hz}, \mathrm{H}-6)\right.$ and $6.56(1 \mathrm{H}, \mathrm{d}, J=$ $2.5 \mathrm{~Hz}, \mathrm{H}-8)]$, a 1,2,3,4-tetrasubstituted benzene ring [ $\delta_{\mathrm{H}} 6.77\left(1 \mathrm{H}, \mathrm{d}, J=8.7 \mathrm{~Hz}, \mathrm{H}-6^{\prime}\right)$ and $6.46(1 \mathrm{H}$, $\left.\left.\mathrm{d}, J=8.7 \mathrm{~Hz}, \mathrm{H}-5^{\prime}\right)\right]$, two trans-olefinic protons $\left[\delta_{\mathrm{H}} 7.03\left(1 \mathrm{H}, \mathrm{dq}, J=15.6,6.9 \mathrm{~Hz}, \mathrm{H}-3^{\prime \prime \prime}\right)\right.$ and 5.90 $\left.\left(1 \mathrm{H}, \mathrm{dd}, J=15.6,1.6 \mathrm{~Hz}, \mathrm{H}-2^{\prime \prime \prime}\right)\right]$, two methoxy groups [ $\delta_{\mathrm{H}} 3.81\left(3 \mathrm{H}, \mathrm{s}, 4^{\prime}-\mathrm{OMe}\right)$ and $3.79\left(3 \mathrm{H}, \mathrm{s}, 3^{\prime}-\right.$ OMe $)$, two oxygenated methylenes [ $\delta_{\mathrm{H}} 4.51\left(1 \mathrm{H}, \mathrm{dd}, J=11.8,2.1 \mathrm{~Hz}, \mathrm{H}-6^{\prime \prime} \mathrm{a}\right), 4.22(1 \mathrm{H}, \mathrm{dd}, J=11.8$, $\left.7.5 \mathrm{~Hz}, \mathrm{H}-6^{\prime \prime} \mathrm{b}\right), 4.27(1 \mathrm{H}$, brd, $J=9.9 \mathrm{~Hz}, \mathrm{H}-2 \mathrm{a})$ and $\left.4.02(1 \mathrm{H}, \mathrm{t}, J=9.9 \mathrm{~Hz}, \mathrm{H}-2 \mathrm{~b})\right]$, five oxygenated methines [ $\delta_{\mathrm{H}} 4.81\left(1 \mathrm{H}, \mathrm{d}, J=7.5 \mathrm{~Hz}, \mathrm{H}-1^{\prime \prime}\right), 3.66\left(1 \mathrm{H}, \mathrm{m}, \mathrm{H}-5^{\prime \prime}\right), 3.46\left(1 \mathrm{H}, \mathrm{m}, \mathrm{H}-3^{\prime \prime}\right), 3.45(1 \mathrm{H}, \mathrm{m}, \mathrm{H}-$ $\left.2^{\prime \prime}\right)$ and $\left.3.35\left(1 \mathrm{H}, \mathrm{m}, \mathrm{H}-4^{\prime \prime}\right)\right]$, a methyl group $\left[\delta_{\mathrm{H}} 1.85\left(3 \mathrm{H}, \mathrm{dd}, J=6.9,1.6 \mathrm{~Hz}, \mathrm{Me}-4^{\prime \prime \prime}\right)\right]$. The ${ }^{13} \mathrm{C}$ NMR spectrum of 1 showed 27 carbon signals, which can be classified by DEPT and HSQC spectra as a carbonyl $\left(\delta_{\mathrm{C}} 168.0\right)$, two benzene rings $\left(\delta_{\mathrm{C}} 158.3,156.4,153.2,149.6,137.6,131.1,122.9,122.3\right.$, $117.8,110.6,105.6$ and 104.4), a disubstituted double bond ( $\delta_{\mathrm{C}} 146.9$ and 123.2$)$, a hexosyl group $\left(\delta_{\mathrm{C}}\right.$ $102.5,77.9,75.5,74.9,71.9$ and 64.7$)$, two methoxy groups $\left(\delta_{\mathrm{C}} 61.6\right.$ and 56.3$)$, two sp ${ }^{3}$ methylenes (one oxygenated at $\delta_{\mathrm{C}} 71.1$ ), a $\mathrm{sp}^{3}$ methine and a methyl. The aforementioned spectroscopic data suggested that compound 1 was an isoflavan glucoside.

The 2D structure of 1 was determined by analysis of its 2D NMR data (Figure S1). The ${ }^{1} \mathrm{H}-{ }^{1} \mathrm{H}$ COSY correlations of $\mathrm{H}_{2}-2 / \mathrm{H}-3 / \mathrm{H}_{2}-4, \mathrm{H}-5 / \mathrm{H}-6, \mathrm{H}-5^{\prime} / \mathrm{H}-6^{\prime}$, in combination with the HMBC correlations of $\mathrm{H}_{2}-2 / \mathrm{C}-9$; $\mathrm{H}_{2}-4 / \mathrm{C}-5$, C-9 and C-10; H-5/C-7 and C-9; H-8/C-6; H-5'/C-3'; H-6'/C-3, C- 
$2^{\prime}$ and C-4' demonstrated the existence of 7,2',3',4'-tetrahydroxyisoflavan moiety. The HMBC correlations from $3^{\prime}-\mathrm{OMe}\left(\delta_{\mathrm{H}} 3.79\right)$ to $\mathrm{C}-3^{\prime}$ and from $4^{\prime}-\mathrm{OMe}\left(\delta_{\mathrm{H}} 3.81\right)$ to $\mathrm{C}-4^{\prime}$ suggested that the methoxy groups were attached to $\mathrm{C}-3^{\prime}$ and $\mathrm{C}-4^{\prime}$, respectively. The hexosyl group was determined to be a $\beta$ glucose by comparison of its $1 \mathrm{D}$ NMR data and coupling constant $\left(J_{1^{\prime \prime} / 2^{\prime \prime}}=7.5\right)$ with reported data [12]. This glucosyl was located at C-7 of the isoflavan moiety as indicated by the HMBC correlation from the anomeric proton $\left(\delta_{\mathrm{H}} 4.81, \mathrm{H}-1^{\prime \prime}\right)$ to the $\mathrm{C}-7\left(\delta_{\mathrm{C}} 158.3\right)$. The ${ }^{1} \mathrm{H}-{ }^{1} \mathrm{H}$ COSY correlations of $\mathrm{H}-$ $2^{\prime \prime \prime} / \mathrm{H}-3^{\prime \prime \prime} / \mathrm{H}_{3}-4^{\prime \prime \prime}$ and HMBC correlation of $\mathrm{H}-3^{\prime \prime \prime} / \mathrm{C}-1^{\prime \prime \prime}$ suggested the presence of a $(E)$-but-2-enoyl group, and this group was linked to $6^{\prime \prime}-\mathrm{OH}$ by $\mathrm{HMBC}$ from $\mathrm{H}_{2}-6^{\prime \prime}$ to $\mathrm{C}-1^{\prime \prime \prime}$. Thus, the planar structure of compound 1 was determined.

The absolute configuration of $\mathbf{1}$ was confirmed by chemical transformation. The alkaline hydrolysis of 1 yielded a known product, $(3 R)-(-)-7,2^{\prime}$-dihydroxy-3',4'-dimethylisoflavan-7- $O-\beta$-Dglucopyranoside (1a), whose absolute configuration was determined by single-crystal X-ray diffraction [with a Flack parameter of 0.05(5)] (Figure 2). Thus, the structure of compound 1 was established as depicted and named astramemside A.

Table 1. ${ }^{1} \mathrm{H}(500 \mathrm{MHz})$ and ${ }^{13} \mathrm{C}(125 \mathrm{MHz}) \mathrm{NMR}$ data of $\mathbf{1}\left(\delta\right.$ in ppm) in $\mathrm{CD}_{3} \mathrm{OD}$

\begin{tabular}{|c|c|c|c|c|c|}
\hline No. & $\delta_{\mathrm{H}}(J$ in $\mathrm{Hz})$ & $\delta \mathrm{C}$ & No. & $\delta_{\mathrm{H}}(J$ in $\mathrm{Hz})$ & $\delta \mathrm{C}$ \\
\hline $2 a$ & 4.27, brd $(9.9)$ & 71.1 & $5^{\prime}$ & $6.46, \mathrm{~d}(8.7)$ & 104.4 \\
\hline $2 b$ & $4.02, \mathrm{t}(9.9)$ & & $6^{\prime}$ & $6.77, \mathrm{~d}(8.7)$ & 122.9 \\
\hline 3 & $3.48, \mathrm{~m}$ & 33.4 & $1^{\prime \prime}$ & $4.81, \mathrm{~d}(7.5)$ & 102.5 \\
\hline \multirow[t]{2}{*}{4} & a $3.00, \mathrm{dd}(15.8,10.4)$ & 31.2 & $2^{\prime \prime}$ & $3.45, \mathrm{~m}$ & 74.9 \\
\hline & b 2.86 , dd $(15.8,4.5)$ & & $3^{\prime \prime}$ & $3.46, \mathrm{~m}$ & 77.9 \\
\hline 5 & $6.97, \mathrm{~d}(8.3)$ & 131.1 & $4^{\prime \prime}$ & $3.35, \mathrm{~m}$ & 71.9 \\
\hline 6 & $6.59, \mathrm{dd}(8.3,2.5)$ & 110.6 & $5^{\prime \prime}$ & $3.66, \mathrm{~m}$ & 75.5 \\
\hline 7 & & 158.3 & $6^{\prime \prime} \mathrm{a}$ & $4.51, \mathrm{dd}(11.8,2.1)$ & 64.7 \\
\hline 8 & $6.56, \mathrm{~d}(2.5)$ & 105.6 & $6^{\prime \prime} \mathrm{b}$ & $4.22, \mathrm{dd}(11.8,7.5)$ & \\
\hline 9 & & 156.4 & $1^{\prime \prime \prime}$ & & 168.0 \\
\hline 10 & & 117.8 & $2^{\prime \prime \prime}$ & $5.90, \mathrm{dd}(15.6,1.6)$ & 123.2 \\
\hline $1^{\prime}$ & & 122.3 & $3^{\prime \prime \prime}$ & $7.03, \mathrm{dq}(15.6,6.9)$ & 146.9 \\
\hline $2^{\prime}$ & & 149.6 & $4^{\prime \prime \prime}$ & $1.85, \mathrm{dd},(6.9,1.6)$ & 18.2 \\
\hline $3^{\prime}$ & & 137.6 & 3'-OMe & $3.79, \mathrm{~s}$ & 61.1 \\
\hline $4^{\prime}$ & & 153.2 & $4^{\prime}-\mathrm{OMe}$ & $3.81, \mathrm{~s}$ & 56.3 \\
\hline
\end{tabular}
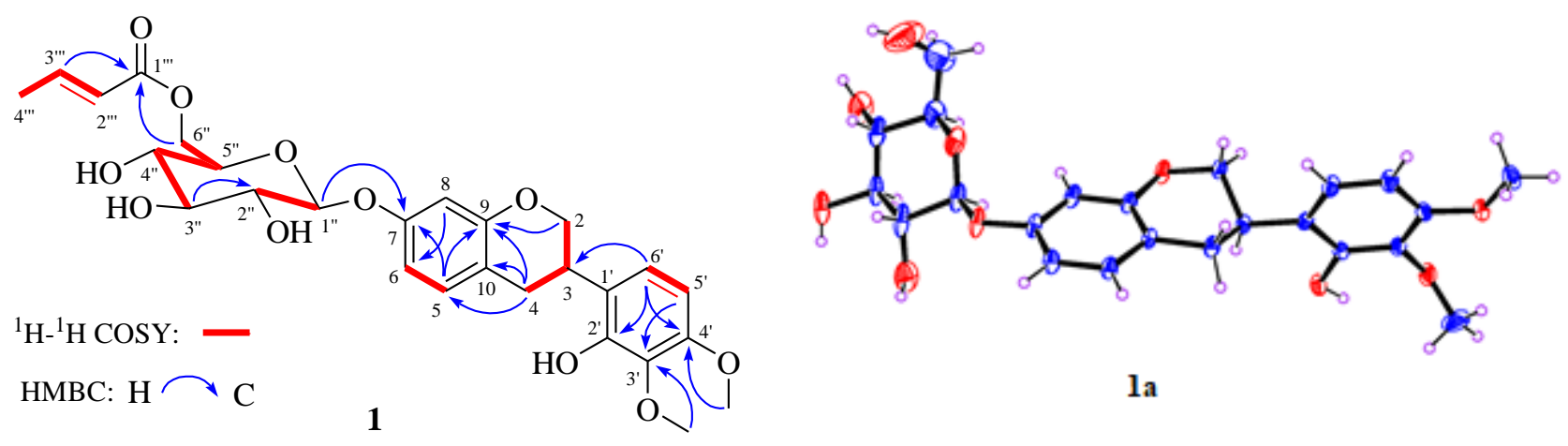

la

Figure 2. Key ${ }^{1} \mathrm{H}-{ }^{1} \mathrm{H}$ COSY and $\mathrm{HMBC}$ correlations of $\mathbf{1}$ (left); X-ray structure of 1a (right) 
The known compounds were identified as 7-O- $\beta$-D-glucopyranosyl 7,3'-dihydroxy-4'-methoxy isoflavone (2) [13], $(6 \mathrm{a} R, 11 \mathrm{a} R)-9,10$-dimethoxypterocarpan-3-O- $\beta$-D-glucoside (3) [14], calycosin (4) [15], isomucronulatol (5) [16], $(6 \mathrm{a} R, 11 \mathrm{a} R)$-10-hydroxy-3,9-dimethoxypterocarpan (6) [17], and 3'hydroxydaidzein (7) [18], by comparison of their spectroscopic data with the published data.

Most of these co-isolated known compounds have been previously reported to possess inhibitory activity on the nitric oxide (NO) production. Among them, compounds $\mathbf{2}$ and $\mathbf{3}$ showed strong inhibitory activity, with with $\mathrm{IC}_{50}$ values of $4.10 \pm 0.10$ and $14.70 \pm 0.90 \mu \mathrm{M}$, respectively [19]. Compound 4 had moderate inhibitory activity $\left(\mathrm{IC}_{50}=39.56 \pm 2.43 \mu \mathrm{M}\right)$, while compound 7 was inactive ( $\mathrm{IC}_{50}>100 \mu \mathrm{M}[20]$. The new compound 1 was tested for its inhibitory effect on the NO production induced by lipopolysaccharide in RAW264.7 cells. Quercetin was used as a positive control $\left(\mathrm{IC}_{50}=17.86 \pm 2.13 \mu \mathrm{M}\right)$. The result showed that compound 1 showed moderate inhibitory activity $\left(\mathrm{IC}_{50}=38.98 \pm 5.28 \mu \mathrm{M}\right)$.

\section{Acknowledgments}

This work was financially supported by the National Key R\&D Program of China (grant number 2020YFC1712700).

\section{Supporting Information}

Supporting Information accompanies this paper on http://www.acgpubs.org/journal/recordsof-natural-products

\section{ORCID}

Yadong Wang: 0000-0002-8815-6397

Chao Wang: 0000-0002-6671-7050

Yuanhao Wang: 0000-0003-4135-7359

Qing Hu: 0000-0002-6532-9718

Fenghuan Wang: 0000-0003-2498-3673

\section{References}

[1] Editor Committee for Flora of China of Chinese Academy of Science (1997). Science Publishing House, Beijing. PP. 133.

[2] X. Li, L. Qu, Y. Dong, L. Han, E. Liu, S. Fang, Y. Zhang and Tao Wang (2014). A review of recent research progress on the Astragalus genus, Molecules 19, 18850-18880.

[3] X. Du, B. Zhao, J. Li, X. Cao, M. Diao, H. Feng, X. Chen, Z. Chen and X. Zeng (2012). Astragalus polysaccharides enhance immune responses of HBV DNA vaccination via promoting the dendritic cell maturation and suppressing Treg frequency in mice, Int. Immunopharmacol. 14, 463-470.

[4] Y. Zhang, X. Li, J. Ruan, T. Wang, Y. Dong, J. Hao, E. Liu, L. Han, X. Gao and T. Wang (2016). Oleanane type saponins from the stems of Astragalus membranaceus (Fisch.) Bge. var. mongholicus (Bge.) Hsiao, Fitoterapia 109, 99-105.

[5] Y. Pei, R. Li, H. Fu, J. Wang and Y. Zhou (2007). A new isoflavone glucoside from Astragalus membranaceus var. mongholicus, Fitoterapia 78, 602-604.

[6] L. M. Xiao, P. H. Cao, Z. H. Luo, X. F. Bao, Z. Q. Zhou, S. Li, S. S. Zhao, J. X. Zheng, H. Gao and H. Zhi (2020). Cycloartane-type triterpenoids from the root of Astragalus membranaceus var. Mongholicus, J. Asian Nat. Prod. Res. 22, 905-913.

[7] Z. Q. He and J. A. Findlay (1991). Constituents of Astragalus membranaceus, J. Nat. Prod. 54, 810815.

[8] Q. H. Wang, N. R. C. K. T. Han, N. Y. T. Dai, X. L. Wang and W. L. J. Ao (2014). Anti-inflammatory effects and structure elucidation of two new compounds from Astragalus membranaceus (Fisch) Bge. var. mongholicus (Bge) Hsiao, J. Mol. Struct. 1074, 284-288. 
[9] X. Du, Y. Bai, H. Liang, Z. Wang, Y. Zhao, Q. Zhang and L. Huang (2006). Solvent effect in ${ }^{1} \mathrm{H}$ NMR spectra of 3'-hydroxy-4'-methoxy isoflavonoids from Astragalus membranaceus var. mongholicus, Magn. Reson. Chem. 44, 708-712.

[10] Q. T. Yu, P. Li, Z. M. Bi, J. Luo and X. D. Gao (2007). Two new saponins from the aerial part of Astragalus membranaceus var. mongholicus, Chin. Chem. Lett. 18, 554-556.

[11] D. Y. Lee, H. J. Noh, J. Choi, K. H. Lee, M. H Lee, J. H. Lee, Y. Hong, S. E. Lee, S. Y. Kim and G. S. Kim (2013). Anti-inflammatory cycloartane-type saponins of Astragalus membranaceus, Molecules $\mathbf{1 8}$, 3725-3732.

[12] L. J. Zhang, H. K. Liu, P. C. Hsiao, L. M. Y. Kuo, I. J. Lee, T. S. Wu, W. F. Chiou and Y. H. Kuo (2011). New isoflavonoid glycosides and related constituents from Astragali Radix (Astragalus membranaceus) and their inhibitory activity on nitric oxide production, J. Agric. Food Chem. 59, 1131-1137.

[13] N. I. Baek, Y. S. Kim, J. S. Kyung and K. H. Park (1996). Isolation of anti-hepatotoxic agent from the root of Astragalus membranaceus, Korean J. Pharmacogn. 27, 111-116.

[14] J. Sun, M. Zhao, H. Liang and P. Tu (2010). Isolation and identification of flavonoids from Baoyuan Decoction, Chin. Tradit. Herb. Drugs 41, 696-700.

[15] R. Y. Yang, Y. S. Lan, Z. J. Huang, C. L. Shao, H. Liang, Z. F. Chen and J. Li (2012). Isoflavonoids from Sophora tonkinensis, Chem. Nat. Compd. 48, 674-676.

[16] E. J. Lee, M. H. Yean, H. S. Jung, J. S. Kim and S. S. Kang (2008). Phytochemical studies on Astragalus root (2)-flavonoids and a lignan, Nat. Prod. Sci. 14, 131-137.

[17] X. Dong, Y. Liang, B. Wang and X. Long (2010). Simultaneous separation of four flavonoids and two astragalosides from Radix Astragali by semi-preparative LC, Chromatographia 71, 225-231.

[18] Y. C. Chen, Y. Sugiyama, N. Abe, R. Kuruto-Niwa, R. Nozawa and A. Hirota (2005). DPPH radicalscavenging compounds from dou-chi, a soybean fermented food, Biosci. Biotechnol. Biochem. 69, 9991006.

[19] L. J. Zhang, H. K. Liu, P. C. Hsiao, L. M. Y. Kuo, I. J. Lee, T. S. Wu, W. F. Chiou and Y. H. Kuo (2011). New isoflavonoid glycosides and related constituents from astragali radix (Astragalus membranaceus) and their inhibitory activity on nitric oxide production, J. Agric. Food Chem. 59, 1131-1137.

[20] Y. Song, L. Pan, W. Li, Y. Si, D. Zhou, C. Zheng, X. Hao, X. Jia, Y. Jia, M. Shi, X. Jia, N. Li and Y. Hou (2017). Natural neuro-inflammatory inhibitors from Caragana turfanensis, Bioorg. Med. Chem. Lett. 27, 4765-4769.

\section{A C G \\ publications \\ (c) 2021ACG Publications}

